\title{
Multiplicative Pulsated Fibonacci Sequences Part 2
}

\author{
Alongkot Suvarnamani", Sakunna Koyram \\ Department of Mathematics, Faculty of Science and Technology, Rajamangala University of Technology Thanyaburi (RMUTT), Thailand
}

Copyright (C) 2015 by authors, all rights reserved. Authors agree that this article remains permanently open access under the terms of the Creative Commons Attribution License 4.0 International License

\begin{abstract}
In this paper, we consider the multiplicative pulsated Fibonacci sequences. First, we show the new proof of the explicit formulas of multiplicative pulsated Fibonacci sequences of second order. Then the second type of multiplicative pulsated Fibonacci sequences is introduced and explicit formulas for the form of its members are formulated and proved.
\end{abstract}

Keywords Fibonacci Sequence, Coupled Fibonacci Sequences, 2-Fibonacci Sequences, Multiplicative Pulsated Fibonacci Sequences

\section{Introduction}

The Fibonacci sequence $\left\{F_{n}\right\}$ is defined as $\mathrm{F}_{0}=0, \mathrm{~F}_{1}=1$ and $\mathrm{F}_{\mathrm{n}+1}=\mathrm{F}_{\mathrm{n}}+\mathrm{F}_{\mathrm{n}-1}$ for the positive integer $n \geq 1$. The Binet's formulas for Fibonacci numbers are given by $F_{n}=\frac{r_{1}^{n}-r_{2}^{n}}{r_{1}-r_{2}}$ where $r_{1}=\frac{1+\sqrt{5}}{2}$ and $r_{2}=\frac{1-\sqrt{5}}{2}$ are roots of the characteristic equation $r^{2}-r-1=0$.

In last decade, the coupled difference equations or recurrence relations are popularized. They involve two sequences of integers in which the elements of one sequence are part of the generalization of the other, and vice versa. We can say that these are generalization of ordinary recursive sequences and many results can be developed for considering the two sequences are identical.

Atanassov is the first person who introduced the concept of coupled Fibonacci sequences. He also discussed many curious properties and new direction of generalization of Fibonacci sequence in [1], [2], [3] and [5] with his friends. He was defined and studied about four different ways to generate coupled sequences and called them 2-Fibonacci sequence (or 2-F sequences). This was new direction of Fibonacci sequence generalizations.

In 2014, Suvarnamani and Jitjang [7] presented the new ideas in generalization of Fibonacci sequences in the case of one or more sequences. They constructed the multiplicative pulsated Fibonacci sequences of second order.
In this paper, we formulated and proved the explicit formulas of multiplicative pulsated Fibonacci sequences of second order in [7]. And we describe the basic concepts that will be used to construct multiplicative pulsated Fibonacci sequences of second order. Further, we shall describe the fundamental properties.

\section{Multiplicative Pulsated Fibonacci Sequences Part 1}

Let $a$ and $b$ be two fixed real numbers. Let us construct the following two sequences

$$
\begin{gathered}
\alpha_{0}=a, \beta_{0}=b, \\
\alpha_{2 k+1}=\beta_{2 k+1}=\alpha_{2 k} \cdot \beta_{2 k}, \\
\alpha_{2 k+2}=\alpha_{2 k+1} \cdot \beta_{2 k}, \\
\beta_{2 k+2}=\beta_{2 k+1} \cdot \alpha_{2 k},
\end{gathered}
$$

\begin{tabular}{|c|c|c|c|}
\hline$k$ & $\alpha_{k}$ & $\alpha_{k}=\beta_{k}$ & $\beta_{k}$ \\
\hline 0 & $a$ & & $b$ \\
\hline 1 & & $a b$ & \\
\hline 2 & $a b^{2}$ & & $a^{2} b$ \\
\hline 3 & & $a^{3} b^{3}$ & \\
\hline 4 & $a^{5} b^{4}$ & & $a^{4} b^{5}$ \\
\hline 5 & & $a^{9} b^{9}$ & \\
\hline 6 & $a^{13} b^{14}$ & & $a^{14} b^{13}$ \\
\hline 7 & & $a^{27} b^{27}$ & \\
\hline 8 & $a^{41} b^{40}$ & & $a^{40} b^{41}$ \\
\hline 9 & & $a^{81} b^{81}$ & \\
\hline 10 & $a^{121} b^{122}$ & & $a^{122} b^{121}$ \\
\hline 11 & & $a^{243} b^{243}$ & \\
\hline$\vdots$ & $\vdots$ & $\vdots$ & $\vdots$ \\
\hline
\end{tabular}

for the natural number $k \geq 0$. This pair of sequences we call a multiplicative pulsated Fibonacci sequences. The first values of the two sequences are given in the Table 1. 
From Table 1, we get some relations in Theorem 1.

Theorem 1. For every integer number $k \geq 0$,

$$
\begin{aligned}
& \alpha_{2 k+1}=\beta_{2 k+1}=a^{\left(3^{k}\right)} \cdot b^{\left(3^{k}\right)}, \\
& \alpha_{2 k}=a^{\left(\frac{3^{k}+(-1)^{k}}{2}\right)} \cdot b^{\left(\frac{3^{k}+(-1)^{k+1}}{2}\right)}, \\
& \beta_{2 k}=a^{\left(\frac{3^{k}+(-1)^{k+1}}{2}\right)} \cdot b^{\left(\frac{3^{k}+(-1)^{k}}{2}\right)} .
\end{aligned}
$$

Proof. We will prove by mathematical induction. Let $P_{k}$ :

$$
\begin{aligned}
& \alpha_{2 k+1}=\beta_{2 k+1}=a^{\left(3^{k}\right)} \cdot b^{\left(3^{k}\right)}, \\
& \alpha_{2 k}=a^{\left(\frac{3^{k}+(-1)^{k}}{2}\right)} \cdot b^{\left(\frac{3^{k}+(-1)^{k+1}}{2}\right)}, \\
& \beta_{2 k}=a^{\left(\frac{3^{k}+(-1)^{k+1}}{2}\right)} \cdot b^{\left(\frac{3^{k}+(-1)^{k}}{2}\right)},
\end{aligned}
$$

for every integer number $k \geq 0$.

If $k=0$, then

$$
\begin{gathered}
\alpha_{2(1)+1}=\alpha_{2(1)} \cdot \beta_{2(1)} \\
=\alpha_{0} \cdot \beta_{0} \\
=a \cdot b \\
=a^{\left(3^{0}\right)} \cdot b^{\left(3^{0}\right)} \cdot \\
\beta_{2(0)+1}=\alpha_{2(0)} \cdot \beta_{2(0)} \\
=\alpha_{0} \cdot \beta_{0} \\
=a^{2} \cdot b^{\left(3^{0}\right)} \cdot b^{\left(3^{0}\right)} \cdot \\
=a^{(0)}=\alpha_{0} \\
=a^{\left(\frac{3^{0}+(-1)^{0}}{2}\right)} \cdot b^{\left(\frac{3^{0}+(-1)^{0+1}}{2}\right)} \\
=a^{1} \cdot b^{0}
\end{gathered}
$$

$$
\begin{aligned}
\beta_{2(0)} & =\beta_{0} \\
& =b \\
& =a^{0} \cdot b^{1} \\
& =a^{\left(\frac{3^{0}+(-1)^{0+1}}{2}\right)} \cdot b^{\left(\frac{3^{0}+(-1)^{0}}{2}\right)} .
\end{aligned}
$$

Thus $P_{0}$ is true.

Next, we assume that $P_{k}$ is true for some integer number $k \geq 0$, i.e.,

$$
\begin{aligned}
& \alpha_{2 k+1}=\beta_{2 k+1}=a^{\left(3^{k}\right)} \cdot b^{\left(3^{k}\right)}, \\
& \alpha_{2 k}=a^{\left(\frac{3^{k}+(-1)^{k}}{2}\right)} \cdot b^{\left(\frac{3^{k}+(-1)^{k+1}}{2}\right)}, \\
& \beta_{2 k}=a^{\left(\frac{3^{k}+(-1)^{k+1}}{2}\right)} \cdot b^{\left(\frac{3^{k}+(-1)^{k}}{2}\right)} .
\end{aligned}
$$

Then we will show that $P_{k+1}$ is true.

$$
\begin{aligned}
\alpha_{2(k+1)+1} & =\beta_{2(k+1)+1} \\
& =\alpha_{2(k+1)} \cdot \beta_{2(k+1)} \\
& =\alpha_{2 k+2} \cdot \beta_{2 k+2} \\
& =\alpha_{2 k+1} \cdot \beta_{2 k} \cdot \beta_{2 k+1} \cdot \alpha_{2 k} \\
& =\alpha_{2 k+1} \cdot \beta_{2 k+1} \cdot\left(\alpha_{2 k} \cdot \beta_{2 k}\right) \\
& =\alpha_{2 k+1} \cdot \beta_{2 k+1} \cdot \alpha_{2 k+1} \\
& =\alpha_{2 k+1} \cdot \alpha_{2 k+1} \cdot \alpha_{2 k+1} \\
& =\left(\alpha_{2 k+1}\right)^{3} \\
& =\left(a^{\left(3^{k}\right)} b^{\left(3^{k}\right)}\right)^{3} \\
& =a^{\left(3 \cdot 3^{k}\right)} \cdot b^{\left(3 \cdot 3^{k}\right)} \\
& =a^{\left(3^{k+1}\right)} \cdot b^{\left(3^{k+1}\right)} \cdot
\end{aligned}
$$




$$
\begin{aligned}
& \alpha_{2(k+1)}=\alpha_{2 k+2} \\
& =\alpha_{2 k+1} \cdot \beta_{2 k} \\
& =\alpha_{2 k} \cdot \beta_{2 k} \cdot \beta_{2 k} \\
& =a^{\left(\frac{3^{k}+(-1)^{k}}{2}\right)} \cdot b^{\left(\frac{3^{k}+(-1)^{k+1}}{2}\right)} \cdot a^{\left(\frac{3^{k}+(-1)^{k+1}}{2}\right)} \cdot b^{\left(\frac{3^{k}+(-1)^{k}}{2}\right)} \cdot a^{\left(\frac{3^{k}+(-1)^{k+1}}{2}\right)} \cdot b^{\left(\frac{3^{k}+(-1)^{k}}{2}\right)} \\
& =a^{\left(\frac{3 \cdot 3^{k}+(-1)^{k+1}}{2}\right)} \cdot b^{\left(\frac{3 \cdot 3^{k}+(-1)^{k}}{2}\right)} \\
& =a^{\left(\frac{3^{k+1}+(-1)^{k+1}}{2}\right)} \cdot b^{\left(\frac{3^{k+1}+(-1)^{(k+1)+1}}{2}\right)} \text {. } \\
& \beta_{2(k+1)}=\beta_{2 k+2} \\
& =\beta_{2 k+1} \cdot \alpha_{2 k} \\
& =\alpha_{2 k} \cdot \beta_{2 k} \cdot \alpha_{2 k} \\
& =a^{\left(\frac{3^{k}+(-1)^{k}}{2}\right)} \cdot b^{\left(\frac{3^{k}+(-1)^{k+1}}{2}\right)} \cdot a^{\left(\frac{3^{k}+(-1)^{k+1}}{2}\right)} \cdot b^{\left(\frac{3^{k}+(-1)^{k}}{2}\right)} \cdot a^{\left(\frac{3^{k}+(-1)^{k}}{2}\right)} \cdot b^{\left(\frac{3^{k}+(-1)^{k+1}}{2}\right)} \\
& =a^{\left(\frac{3^{k+1}+(-1)^{(k+1)+1}}{2}\right)} \cdot b^{\left(\frac{3^{k+1}+(-1)^{k+1}}{2}\right)} \text {. }
\end{aligned}
$$

So, $P_{m+1}$ is true.

By mathematical induction, $\left[P_{k}\right.$ is true for all integer number $k \geq 0$.

\section{Multiplicative Pulsated Fibonacci Sequences Part 2}

Now, a new type of Fibonacci-like sequence is introduced. Let $a, b$ and $c$ be three fixed real numbers. Let us construct the following two sequences

$$
\begin{gathered}
\alpha_{0}=a, \beta_{0}=b, \\
\alpha_{1}=\beta_{1}=c, \\
\alpha_{2 k}=\alpha_{2 k-1} \cdot \beta_{2 k-2}, \\
\beta_{2 k}=\beta_{2 k-1} \cdot \alpha_{2 k-2}, \\
\alpha_{2 k+1}=\beta_{2 k+1}=\alpha_{2 k} \cdot \beta_{2 k},
\end{gathered}
$$

for the natural number $k \geq 0$. This pair of sequences is called a multiplicative pulsated Fibonacci sequences. The first values of the two sequences are given in the Table 2.
Table 2. The Multiplicative Pulsated Fibonacci Sequences Part 2

\begin{tabular}{|c|c|c|c|}
\hline$k$ & $\alpha_{k}$ & $\alpha_{k}=\beta_{k}$ & $\beta_{k}$ \\
\hline 0 & $a$ & $c$ & $b$ \\
1 & & & \\
2 & $b c$ & $a b c^{2}$ & $a c$ \\
3 & & & $a b^{2} c^{3}$ \\
4 & $a^{2} b c^{3}$ & $a^{3} b^{3} c^{6}$ & \\
5 & & & $a^{5} b^{4} c^{9}$ \\
6 & $a^{4} b^{5} c^{9}$ & $a^{9} b^{9} c^{18}$ & \\
7 & & $a^{13} b^{14} c^{27}$ \\
8 & $a^{14} b^{13} c^{27}$ & & $a^{27} b^{27} c^{54}$ \\
9 & & $\vdots$ & $a^{41} b^{40} c^{27}$ \\
10 & $a^{40} b^{41} c^{81}$ & $\vdots$ \\
$\vdots$ & $\vdots$ & $\vdots$ & $\vdots$ \\
\hline
\end{tabular}


From Table 2, we get some relations in Theorem 2 .

Theorem 2. For every integer number $k \geq 1$,

$$
\begin{gathered}
\alpha_{2 k}=a^{\left(\frac{3^{k-1}+(-1)^{k}}{2}\right)} \cdot b^{\left(\frac{3^{k-1}+(-1)^{k-1}}{2}\right)} \cdot c^{\left(3^{k-1}\right)}, \\
\beta_{2 k}=a^{\left(\frac{3^{k-1}+(-1)^{k-1}}{2}\right)} \cdot b^{\left(\frac{3^{k-1}+(-1)^{k}}{2}\right)} \cdot c^{\left(3^{k-1}\right)}, \\
\alpha_{2 k+1}=\beta_{2 k+1}=a^{\left(3^{k-1}\right)} \cdot b^{\left(3^{k-1}\right)} \cdot c^{2 \cdot\left(3^{k-1}\right)} \cdot
\end{gathered}
$$

Proof.

We will use the mathematical induction.

Let $P_{k}$ :

$$
\begin{gathered}
\alpha_{2 k}=a^{\left(\frac{3^{k-1}+(-1)^{k}}{2}\right)} \cdot b^{\left(\frac{3^{k-1}+(-1)^{k-1}}{2}\right)} \cdot c^{\left(3^{k-1}\right)} \\
\beta_{2 k}=a^{\left(\frac{3^{k-1}+(-1)^{k-1}}{2}\right)} \cdot b^{\left(\frac{3^{k-1}+(-1)^{k}}{2}\right)} \cdot c^{\left(3^{k-1}\right)} \\
\alpha_{2 k+1}=\beta_{2 k+1}=a^{\left(3^{k-1}\right)} \cdot b^{\left(3^{k-1}\right)} \cdot c^{2 \cdot\left(3^{k-1}\right)} \cdot
\end{gathered}
$$

for every integer number $k \geq 1$.

If $k=1$, then

$$
\begin{aligned}
\alpha_{2(1)} & =\alpha_{2} \\
& =b \cdot c \\
& =a^{\left(\frac{3^{1-1}-1}{2}\right)} \cdot b^{\left(\frac{3^{1-1}+1}{2}\right)} \cdot c^{\left(3^{0}\right)} \cdot \\
\beta_{2(1)} & =\beta_{2} \\
& =a \cdot c \\
& =a^{\left(\frac{3^{1-1}+1}{2}\right)} \cdot b^{\left(\frac{3^{1-1}-1}{2}\right)} \cdot c^{\left(3^{0}\right)} . \\
\alpha_{2(1)+1} & =\alpha_{3} \\
& =a \cdot b \cdot c^{2} \\
& =a^{\left(3^{1-1}\right)} \cdot b^{\left(3^{1-1}\right)} \cdot c^{2 \cdot\left(3^{1-1}\right)} . \\
\beta_{2(1)+1} & =\beta_{3} \\
& =a \cdot b \cdot c^{2} \\
& =a^{\left(3^{1-1}\right)} \cdot b^{\left(3^{1-1}\right)} \cdot c^{2 \cdot\left(3^{1-1}\right)} .
\end{aligned}
$$

Next, we assume that $\mathrm{P}_{k}$ is true for some $k \geq 1$ i.e.,

$$
\begin{gathered}
\alpha_{2 k}=a^{\left(\frac{3^{k-1}+(-1)^{k}}{2}\right)} \cdot b^{\left(\frac{3^{k-1}+(-1)^{k-1}}{2}\right)} \cdot c^{\left(3^{k-1}\right)}, \\
\beta_{2 k}=a^{\left(\frac{3^{k-1}+(-1)^{k-1}}{2}\right)} \cdot b^{\left(\frac{3^{k-1}+(-1)^{k}}{2}\right)} \cdot c^{\left(3^{k-1}\right)}, \\
\alpha_{2 k+1}=\beta_{2 k+1}=a^{\left(3^{k-1}\right)} \cdot b^{\left(3^{k-1}\right)} \cdot c^{2 \cdot\left(3^{k-1}\right)} \cdot
\end{gathered}
$$

To show that $\mathrm{P}_{k+1}$ is true.

$$
\begin{aligned}
\alpha_{2(k+1)} & =\alpha_{2 k+2} \\
& =\alpha_{2 k+1} \cdot \beta_{2 k} \\
& =\alpha_{2 k} \cdot \beta_{2 k} \cdot \beta_{2 k} \\
& =a^{\left(\frac{3 \cdot 3^{k-1}+(-1)^{k-1}}{2}\right)} \cdot b^{\left(\frac{3 \cdot 3^{k-1}+(-1)^{k}}{2}\right)} \cdot c^{\left(3 \cdot 3^{k-1}\right)} \\
& =a^{\left(\frac{3^{(k+1)-1}+(-1)^{k+1}}{2}\right)} \cdot b^{\left(\frac{3^{(k+1)-1}+(-1)^{(k+1)-1}}{2}\right)} \cdot c^{\left(3^{(k+1)-1}\right)} .
\end{aligned}
$$$$
\beta_{2(k+1)}=\beta_{2 k+2}
$$$$
=\beta_{2 k+1} \cdot \alpha_{2 k}
$$$$
=\alpha_{2 k} \cdot \beta_{2 k} \cdot \alpha_{2 k}
$$$$
=a^{\left(\frac{3 \cdot 3^{k-1}+(-1)^{k}}{2}\right)} \cdot b^{\left(\frac{3 \cdot 3^{k-1}+(-1)^{k+1}}{2}\right)} \cdot c^{\left(3 \cdot 3^{k-1}\right)}
$$$$
=a^{\left(\frac{3^{(k+1)-1}+(-1)^{(k+1)-1}}{2}\right)} \cdot b^{\left(\frac{3^{(k+1)-1}+(-1)^{k+1}}{2}\right)} \cdot c^{\left(3^{(k+1)-1}\right)} .
$$$$
\alpha_{2(k+1)+1}=\alpha_{2 k+3}
$$$$
=\alpha_{2 k+2} \cdot \beta_{2 k+2}
$$$$
=\alpha_{2(k+1)} \cdot \beta_{2(k+1)}
$$$$
=a^{\left(\frac{2 \cdot 3^{(k+1)-1}}{2}\right)} \cdot b^{\left(\frac{2 \cdot 3^{(k+1)-1}}{2}\right)} \cdot c^{\left(2 \cdot 3^{(k+1)-1}\right)}
$$$$
=a^{\left(3^{(k+1)-1}\right)} \cdot b^{\left(3^{(k+1)-1}\right)} \cdot c^{2 \cdot\left(3^{(k+1)-1}\right)} \text {. }
$$$$
\beta_{2(k+1)+1}=\beta_{2 k+3}
$$$$
=\alpha_{2 k+2} \cdot \beta_{2 k+2}
$$$$
=\alpha_{2(k+1)} \cdot \beta_{2(k+1)}
$$$$
=a^{\left(\frac{2 \cdot 3^{(k+1)-1}}{2}\right)} \cdot b^{\left(\frac{2 \cdot 3^{(k+1)-1}}{2}\right)} \cdot c^{\left(2 \cdot 3^{(k+1)-1}\right)}
$$$$
=a^{\left(3^{(k+1)-1}\right)} \cdot b^{\left(3^{(k+1)-1}\right)} \cdot c^{2 \cdot\left(3^{(k+1)-1}\right)} \text {. }
$$ 
So, $\mathbf{P}_{k+1}$ is true.

By mathematical induction, $\mathrm{P}_{k}$ is true for all integer $k \geq 1$.

\section{Conclusions}

In this study, we have two points. First, we show the new proof of the explicit formulas of multiplicative pulsated Fibonacci sequences of second order. That is

$$
\begin{aligned}
& \alpha_{2 k+1}=\beta_{2 k+1}=a^{\left(3^{k}\right)} \cdot b^{\left(3^{k}\right)}, \\
& \alpha_{2 k}=a^{\left(\frac{3^{k}+(-1)^{k}}{2}\right)} \cdot b^{\left(\frac{3^{k}+(-1)^{k+1}}{2}\right)}, \\
& \beta_{2 k}=a^{\left(\frac{3^{k}+(-1)^{k+1}}{2}\right)} \cdot b^{\left(\frac{3^{k}+(-1)^{k}}{2}\right)} .
\end{aligned}
$$

for every integer number $k \geq 0$.

Second, we introduced the second type of multiplicative pulsated Fibonacci sequences and proved explicit formulas for the form of its members. We have

$$
\begin{gathered}
\alpha_{2 k}=a^{\left(\frac{3^{k-1}+(-1)^{k}}{2}\right)} \cdot b^{\left(\frac{3^{k-1}+(-1)^{k-1}}{2}\right)} \cdot c^{\left(3^{k-1}\right)}, \\
\beta_{2 k}=a^{\left(\frac{3^{k-1}+(-1)^{k-1}}{2}\right)} \cdot b^{\left(\frac{3^{k-1}+(-1)^{k}}{2}\right)} \cdot c^{\left(3^{k-1}\right)}, \\
\alpha_{2 k+1}=\beta_{2 k+1}=a^{\left(3^{k-1}\right)} \cdot b^{\left(3^{k-1}\right)} \cdot c^{2 \cdot\left(3^{k-1}\right)} \cdot
\end{gathered}
$$

for every integer number $k \geq 0$,

\section{Acknowledgements}

This research was partly supported by Department of Mathematics, Faculty of Science and Technology, Rajamangala University of Technology Thanyaburi (RMUTT), Pathum Thani, THAILAND.

\section{REFERENCES}

[1] Atanassov, K. T., On a Second New Generalization of the Fibonacci Sequence, The Fibonacci Quarterly, Vol. 24, No. 4, (1986), 362-365.

[2] Atanassov, K. T., Remark on a New Direction for a Generalization of the Fibonacci Sequence, The Fibonacci Quarterly, Vol. 33, No. 3, (1995), 249-250.

[3] Atanassov, K. T., Atanassov, L. C. and Sasselov, D. D., A New Perspective to the Generalization of the Fibonacci Sequence, The Fibonacci Quarterly, Vol. 23, No. 1, (1985), 21-28.

[4] Atanassov, K. T., Atanassov, V., Shannon, A. G. and Turner, J. C., New Visual Perspectives on Fibonacci Numbers, World Scientific 2002.

[5] Atanassov, K. T., Pulsating Fibonacci sequences, Notes on Number Theory and Discrete Mathematics, Vol. 19, 2013, No. $3,12-14$.

[6] Lee, J. Z. and Lee, J. S., Some Properties of the Generalization of the Fibonacci Sequence, The Fibonacci Quarterly, Vol. 25, No. 2, (1987), 111-117.

[7] Suvarnamani, A. and Jitjang, S., Multiplicative Pulsated Fibonacci Sequence, Proceeding of ICCSCM 2014, CD-Format, Malaysia, 8-9 May 2014. 